\title{
La doctrine du nombre parfait dans une glose médiévale sur Martianus Capella
}

Jean-Yves Guillaumin

\section{(2) OpenEdition}

1 Journals

Édition électronique

URL : https://journals.openedition.org/philosant/2244

DOI : $10.4000 /$ philosant.2244

ISSN : 2648-2789

Éditeur

Éditions Vrin

\section{Édition imprimée}

Date de publication : 30 octobre 2010

Pagination : 167-184

ISBN : 978-2-7574-0179-8

ISSN : 1634-4561

\section{Référence électronique}

Jean-Yves Guillaumin, « La doctrine du nombre parfait dans une glose médiévale sur Martianus Capella », Philosophie antique [En ligne], 10 | 2010, mis en ligne le 11 juillet 2019, consulté le 02 décembre 2022. URL : http://journals.openedition.org/philosant/2244 ; DOI : https://doi.org/10.4000/ philosant.2244

\section{(c) (†) $\odot$}

Creative Commons - Attribution - Pas d'Utilisation Commerciale - Pas de Modification 4.0 International - CC BY-NC-ND 4.0

https://creativecommons.org/licenses/by-nc-nd/4.0/ 


\section{LA DOCTRINE DU NOMBRE PARFAIT DANS UNE GLOSE MÉDIÉVALE SUR MARTIANUS CAPELLA}

Jean-Yves GUILLAUMIN

Université de Franche-Comté

RÉSUMÉ. Si la doctrine arithmologique des nombres «parfaits », « abondants » et « déficients » est bien connue et souvent exposée depuis Nicomaque de Gérasa (II ${ }^{\mathrm{e}}$ siècle apr. J.-C.), elle reçoit une illustration nouvelle, dans laquelle la mythologie cède le pas à l'éthique et à la théologie, dans une glose médiévale du texte de Martianus Capella (livre VII des Noces de Philologie et de Mercure), où le nombre parfait est mis en correspondance avec la justice éternelle.

SUMMARY. Though widely known and frequently put forward since Nicomachus of Gerasa (2nd century AD), the arithmological doctrine of "perfect", "abundant", and "deficient" numbers is newly illustrated in a medieval gloss to Martianus Capella's De Nuptiis Philologiae et Mercurii, Book. VII, where mythology is replaced by ethics and theology, and the perfect number is compared to eternal justice. 



\section{Introduction : textes mathématiques classiques et textes médiévaux}

Sans doute la mathématique grecque ${ }^{1}$ est-elle fondatrice de tous les développements de cette science sur les bases établies par Pythagore peut-être, par Platon sûrement, puis par les Alexandrins et plusieurs autres. Sans doute a-t-elle, à ce titre, une prééminence indiscutée sur ses avatars postérieurs, romains, tardo-antiques et médiévaux. Et cependant, dans la mesure même où ils témoignent de l'extraordinaire vitalité de la science grecque à travers ses multiples transformations, textes d'époque romaine ${ }^{2}$ ou plus tardive et même textes médiévaux de mathématiques doivent se voir reconnaître un intérêt considérable et faire l'objet d'enquêtes qui puissent permettre de suivre, à travers la longue succession des siècles et des mentalités, les permanences et les évolutions dans la pensée mathématique, dans ses formulations et dans les points d'application quelquefois inattendus qu'on peut lui découvrir.

Les champs d'investigation qui s'ouvrent sont nombreux et variés. Ils ne peuvent se restreindre à la destinée même de la grande mathématique, c'est-à-dire à la géométrie grecque des Euclide, des Apollonius et des Archimède. Les grandes synthèses d'époque tardive, dans lesquelles la mathématique occupe un secteur du savoir encyclopédique recommandé, à côté d'un autre ensemble formé par les disciplines "littéraires », constituent un secteur d'enquête tout à fait intéressant ${ }^{3}$. En effet, élaborées à

1. À propos de laquelle on peut renvoyer aux ouvrages de base que sont par exemple celui de Heath 1921 et celui de Michel 1950.

2. On doit indiquer l'existence d'une thèse suédoise récemment soutenue à l'Université de Göteborg (mars 2009) sur la manière dont la géométrie a été considérée et « revisitée » par les Romains : E. Bohlin, Geometry in Cicero, Varro and Vitruvius.

3. Sur la question précise du vocabulaire de la géométrie chez Martianus Capella, il existe une thèse espagnole récente (soutenue à Madrid, Universidad Nacional de Educación a Distancia, en septembre 2008) : M. Ayuso García, La terminología latina de la geometría en Marciano Capela. 
la fin de l'Antiquité sur la base des connaissances accumulées et théorisées depuis des siècles déjà, elles sont devenues la base d'encyclopédies alto-médiévales puis carolingiennes dans lesquelles le Moyen Âge est allé chercher tous les éléments de son éducation et de sa culture. Boèce et Martianus Capella, pour ne citer qu'eux, sont les piliers de l'enseignement médiéval du quadrivium (et du trivium si l'on parle de Martianus, et de la logique aristotélicienne si l'on parle de Boèce). Des commentateurs médiévaux ont commenté ces commentateurs tardo-antiques. Mais ces médiévaux ont pu manifester autant d'indépendance d'esprit et d'intelligence innovatrice qu'en avaient montré leurs modèles, non seulement Boèce dont les qualités intellectuelles n'ont jamais été discutées par personne, mais aussi Martianus qui n'a pas toujours eu le même bonheur.

Les Noces de Pbilologie et de Mercure écrites au Ve siècle par l'Africain Martianus Capella donnaient, sous la forme allégorique d'un récit de mariage auquel étaient consacrés principalement les deux premiers livres, un compendium des sept «disciplines » considérées l'une après l'autre, les trois «littéraires» dans les livres III à V, puis les quatre «mathématiques» dans les livres VI à IX. L'ampleur du projet (non exclusive, du reste, d'une certaine superficialité, compréhensible au regard précisément de l'immensité des données à mettre en jeu) a poussé la postérité à faire des Noces un manuel d'enseignement extrêmement répandu, tout en l'équipant des notes et commentaires qui devaient en rendre l'utilisation plus facile. C'est d'ailleurs l'époque carolingienne qui a apporté la célébrité à un Martianus Capella vraisemblablement ignoré de ses contemporains et des siècles immédiatement postérieurs.

\section{Un commentaire carolingien sur Martianus Capella}

Une partie de ce matériel réflexif et didactique est conservée sous forme de gloses dans les marges de différents manuscrits carolingiens ${ }^{4}$ de Martianus. Ces gloses remontent à un commentaire organisé dont on a sélectionné les éléments qui ont paru plus particulièrement adaptés à la lecture «renseignée » de l'encyclopédie des Noces. Sur l'auteur de ce commentaire, différentes hypothèses se sont succédé, Martin de Laon ayant détrôné Dunchad de cette fonction, avant d'être lui-même contesté et remplacé par un anonyme inconnu5. La question de l'auteur n'est pas celle qui nous parait essentielle. Il est plus important d'examiner les procédés d'élaboration des commentaires et les arrière-plans culturels et

4. Leyde, Universiteitsbibliotheek, Vossianus Latinus Folio 48 ; Besançon, Bibliothèque Municipale, Ms. 594; Leyde, Universiteitsbibliotheek, BPL 88; Cité du Vatican, Biblioteca Apostolica Vaticana, Reg. lat. 1987.

5. Voir Préaux 1953. 
philosophiques sur lesquels ils se profilent. Ainsi pourra-t-on se faire une idée de la manière dont viennent se greffer sur des doctrines très anciennes des considérations qui témoignent de nouveaux modèles de référence et de préoccupations qui n'étaient pas celles de l'Antiquité.

On peut choisir, pour ce genre d'enquête, entre un très grand nombre d'échantillons pris dans le texte du commentaire ; et on doit le faire, puisqu'il serait bien difficile, trop coûteux en ampleur et en temps, de prétendre dominer d'un seul coup un ensemble multiple et pluriforme. Nous nous intéresserons donc, dans cette étude, non pas à la totalité du plus ancien commentaire, d'auteur inconnu, sur l'œuvre de Martianus, mais, à l'intérieur de ce commentaire, à ce qui porte sur le livre VII consacré à l'arithmétique ${ }^{6}$; précisément, à ce que présentent de ce commentaire les gloses conservées dans les manuscrits de Leyde, Vossianus 48, et de Besançon, BM 594 ; et plus précisément encore, à l'enseignement procuré par ces gloses à propos des nombres déficients, abondants et parfaits. Il s'agit en effet d'une question de théorie arithmétique à la fois très ancienne et très simple, mais maintes fois abordée par les différents commentateurs en différentes langues et à différentes époques, et à ce titre miroir intéressant des permanences et des transformations d'une tradition multiséculaire.

\section{Nombres parfaits, abondants et déficients}

La doctrine elle-même se ramène à ce qui suit7 . Parmi les différentes classifications «pythagoriciennes » du nombre, dont la première est celle qui porte répartition entre pair et impair, figure une répartition en « déficients ", « abondants » et " parfaits »; c'est, d'après Boèce ${ }^{8}$, la deuxième classification du nombre pair (numerorum parium secunda diuisio). La dési-

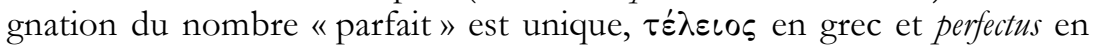
latin; pour les deux autres, les termes peuvent varier dans les deux langues. En tout cas, le nombre " parfait» est celui qui est égal à la somme de ses parties aliquotes ${ }^{9}$; le nombre «déficient» est celui dont la somme des diviseurs reste inférieure au nombre lui-même; le nombre « abondant », au contraire, a des diviseurs dont la somme est supérieure à

6. Ces gloses, telles qu'elles se présentent dans le Vossianus 48, font l'objet d'une entreprise d'édition : voir le site organisé par M. Teeuwen, https://www.e-laborate.nl: 9011/en/carolingian_scholarship/

7. Voir Michel 1950, p. 342 sq.

8. Institution arithmétique, I, 19, 2.

9. Euclide, Éléments, VII, déf. 22 : «Le nombre parfait est celui qui est égal à ses parties. » 
la valeur numérique du nombre lui-même ${ }^{10}$. À propos de ces nombres, les digressions sur la vertu qui tient le juste milieu entre le vice par excès et le vice par défaut ${ }^{11}$ sont compréhensibles et attendues; de fait, on les rencontre d'abord chez Nicomaque ${ }^{12}$ et chez Boèce ${ }^{13}$.

Comme les nombres parfaits, abondants et déficients figurent chez Martianus Capella après avoir été l'objet de l'enseignement de Nicomaque $^{14}$ et avant qu'on ne les retrouve chez Boèce, puis Cassiodore $^{15}$, puis Isidore de Séville ${ }^{16}$, l'auteur du commentaire ancien sur les Noces de Philologie et de Mercure leur consacre un court développement théorique, devenu une glose qui figure en regard du texte de Martianus VII, $\int 753$ (consacré à ces trois subdivisions du nombre pair) au folio 69 recto du manuscrit de Leyde, Vossianus 48 et au folio 119 du manuscrit de Besançon, BM 594. Le texte de cette glose, lu dans le manuscrit de Besançon et dans le manuscrit de Leyde (les insignifiantes erreurs de chacun de ces manuscrits étant corrigées par le texte correct de l'autre), est celui-ci :

Philosophi dicunt omnem virtutem medietatem quandam inter duo vitia tenere; omne namque nitium aut ex superfluo aut ex defectu nascitur; inter superfluum autem et defectum perfectio est; quod nec plus nec minus est, medietatem tenet. Si enim virtus in rationabili animorum motu constat, sequitur ut uitium in motu inrationabili inueniatur. Quod ergo nec ad perfectam rationem contingit uel perfectionem rationis transgreditur uitium est. Inter haec duo motus ipse rationabilis, cui nec aliquid ex superfluo additur, nibil ab eo demitur, perfecta quaedam medietas est, ideoque virtus est. Hac ergo similitudine perfectus numerus dicitur similitudinem nirtutis babere, plus uero quam perfectus uel minus quam perfectus imaginem quandam uitiorum. Verbi gratia, iustitia aeterna, quasi soli Deo seruitur, perfecta est, nibil ex superfluo continet, nibil inperfectionis habet, sicuti est senarius, suis partibus aequalis; bumana uero iustitia, quae saepissime fallit, aut superfluo indicat, ut est duodenarius, quem suae partes superant, aut minus quam oportet indicat, sicut est sedenarius, cui partes propriae aequales non sunt; minorem enim numerum gignunt, id est $\mathrm{XV}$.

10. Aucun de ces deux nombres ne fait l'objet d'une définition chez Euclide. Leur intérêt, en effet, est seulement arithmologique.

11. Cf. Aristote, Étbique à Nicomaque, II, 6, 1106b24 sq.

12. Introduction arithmétique, I, 14, $2 ; \mathrm{I}, 16,1$ et 3.

13. Institution arithmétique, I, 19, 9 ; I, 20, 1 .

14. Et aussi de son contemporain Théon de Smyrne, cf. Expositio rerum mathematicarum, p. 45 Hiller.

15. Institutions, II, $4,4$.

16. Étymologies, III, 5, 9-11. 
L'inscription de la problématique du «nombre parfait» dans le cadre de la réflexion morale sur les vices et les vertus laisse facilement apercevoir ses antécédents. Nicomaque ${ }^{17}$ disait que c'est «dans l'intervalle entre le plus et le moins» que se tiennent «les vertus». Son adaptateur latin Boèce ${ }^{18}$ a caractérisé de façon plus frappante le nombre " parfait» comme uirtutis aemulator. Avant Boèce, Martianus Capella lui-même ${ }^{19}$ se bornait à définir les trois catégories de nombres, sans développements consacrés aux arrière-plans éthiques de la théorie. Le commentateur a trouvé bon de réintroduire cette dimension éthique dans son exposé arithmétique. Il se place ainsi dans le prolongement de Boèce dont il utilise et développe les considérations morales sur cette question.

Ce qui fait le mieux apparaître la tonalité boécienne des phrases consacrées par la glose au rapport de ressemblance entre le nombre parfait et la vertu (et aussi entre le nombre déficient ou abondant et le vice) est précisément l'emploi des termes similitudo et imago, le second étant une variante du premier: la phrase Perfectus numerus dicitur similitudinem uirtutis habere, plus uero quam perfectus uel minus quam perfectus imaginem quandam uitiorum rappelle nettement deux expressions de Boèce : uirtutis aemulator, « imitateur de la vertu », à propos du nombre parfait ${ }^{20}$; Est autem in his quoque magna similitudo uirtutis et uitii, «Il y a dans ces nombres une grande similitude avec le vice et la vertu $»^{21}$.

\section{Définition platonisante du vice et de la vertu}

L'enseignement sur la vertu et sur le vice son contraire entraîne l'exposition de la doctrine des deux mouvements de l'âme, qui n'a rien à voir avec le problème d'arithmétique (ou d'arithmologie) dont traite la glose, et qui laisse d'autant mieux apercevoir dans quelle direction morale le commentateur entend transporter sa matière. Il y a, rappelle-t-il, un mouvement rationnel de l'âme, et un mouvement irrationnel. La doctrine a pour source lointaine l'exposé platonicien sur l'Âme du Monde dans le Timée ${ }^{22}$. Les corps célestes sont entraînés par deux mouvements contraires $^{23}$ selon qu'ils appartiennent à la sphère des fixes, relevant du mouvement du Même, le motus rationalis, ou selon qu'il s'agit des planètes, sou-

17. Introduction arithmétique, I, 14, 2.

18. Institution arithmétique, I, 19, 9.

19. Dans les passages des Noces de Philologie et de Mercure qui font l'objet de nos gloses (VII, 753).

20. Institution arithmétique, I, 19, 9.

21. Institution arithmétique, I, 20, 1.

22. Timée, 34b sq.

23. Timée, 36c-d. 
mises à un motus irrationalis, le mouvement qui relève de l'Autre. Mais ces deux mouvements règlent aussi les comportements et les actions des hommes. Le motus rationalis nous emporte vers l'accomplissement du bien et vers la connaissance du vrai bonheur ; le motus irrationalis nous entraîne dans la direction contraire. Le motus rationalis est donc celui de la vertu, et le «mouvement irrationnel» celui du vice. Ce platonisme médiéval oriente vers les milieux d'Irlandais hellénisants dont fait partie l'auteur du commentaire sur Martianus.

\section{Les exemples}

Les exemples qui sont donnés pour illustrer les notions de nombre parfait, nombre déficient et nombre abondant sont tout à fait canoniques. C'est le cas, en particulier, du nombre 6, que l'on retrouve en cette fonction dans une multitude de textes. Nicomaque ${ }^{24}$ et Boèce ${ }^{25}$, ayant posé que $6=1+2+3$, lui adjoignaient les exemples identiques et complémentaires du nombre $28(=1+2+4+7+14)$ et du nombre 496 ; Ailleurs ${ }^{26}$, ils y avaient ajouté le nombre 8128 . Mais l'exemple consacré et, pourrait-on dire, l'archétype du nombre parfait, reste le nombre 6. Cassiodore se bornait à cet exemple ; Isidore, d'après Boèce évidemment, citait les quatre premiers nombres parfaits. Martianus ${ }^{27}$ ne prenait que l'exemple numérique de 6 et le commentateur, qui n'oublie pas qu'il commente Martianus, fait de même.

Pour le nombre abondant, l'exemple numérique de la glose est 12 , et pour le nombre déficient, 16 . Boèce ${ }^{28}$ ajoutait l'exemple du nombre 24 à celui du nombre 12 pour le nombre abondant. Martianus ${ }^{29}$ se bornait à donner l'exemple de 12 , et le commentateur le suit dans cette brièveté. Les exemples fournis par Boèce ${ }^{30}$ pour le nombre déficient étaient ceux de 8 et de 14 (qu'il empruntait, là encore, sans y rien changer, à son modèle grec Nicomaque ${ }^{31}$ ). Pour le même nombre abondant, la démarche de Martianus était différente : comme il venait d'arriver à un total de 16 pour les diviseurs du nombre 12, modèle de l'«abondant », il reprenait immédiatement ${ }^{32}$ ce nombre 16 pour en faire l'exemple du « déficient». Le commentateur, là encore, suit Marianus, et indique comme exemple

24. Introduction arithmétique, I, 16, 2-3.

25. Institution arithmétique, I, 20, 6-7.

26. Introduction arithmétique, I, 16, 3 ; Institution arithmétique, I, 20, 1.

27. Au $₫ 753$ du livre VII.

28. I, 19, 3 ; suivant là-dessus Nicomaque I, 14, 3 .

29. VII, 753 .

30. I, 19, 6 .

31. I, 14, 1-2.

32. Fin du $\ 753$ du livre VII. 
de nombre déficient le nombre 16 des Noces, non pas les nombres 8 ou 14 de la tradition boécienne. Ce n'est donc pas dans le choix des exemples que le commentateur fait preuve d'originalité; sur ce point, il s'inscrit au contraire dans une tradition qui a toute chance d'être déjà plus que millénaire, même si nous ne la connaissons par des textes que depuis Nicomaque et Théon de Smyrne.

\section{Les illustrations}

Nicomaque et Boèce enrichissaient leurs définitions des nombres abondants, déficients et parfaits d'une série d'illustrations particulièrement suggestives. Celles-ci étaient empruntées à la tradition mythologique ou à celle du Physiologus. Nicomaque ${ }^{33}$ faisait défiler pour illustrer le nombre abondant le monstre Scylla de l'Odyssée ("à dix langues", et à dix gueules $^{34}$ ) et l'Hécatonchire, avec des créatures à neuf lèvres ou trois rangées de dents, ou ayant à la main plus de doigts qu'il n'en faut ; pour le nombre déficient, il alignait ${ }^{35}$ le Cyclope avec son œil unique ${ }^{36}$ et des créatures à un seul bras, moins de cinq doigts à la main, privées de langue ou d'un autre membre. Boèce, à sa suite, convoquait ${ }^{37}$ d'une part l'Hécatonchire avec, innovation de sa part, Géryon aux trois corps, et d'autre part le Cyclope. Même le chrétien Boèce, donc, n’avait pas jugé bon de ne pas se conformer sur ce point à la tradition arithmologique qu'il héritait de Nicomaque. Il manifestait ainsi que son but n'était autre que de permettre, dans l'Italie des années 500, la lecture d'un ouvrage grec de référence dont il n'entendait pas renouveler ni modifier les implications, quoique sa rédaction remontât sans doute à trois siècles et demi. Nicomaque et Boèce ne fournissaient d'exemples mythologiques ou fantastiques que pour le nombre déficient et pour le nombre abondant, sans en donner pour le nombre parfait. Martianus (ce qui ne manque pas de surprendre chez lui, qui est assez porté au fabuleux), Cassiodore et Isidore n'offraient aucune illustration, pour aucune de ces trois classes du nombre pair.

L'auteur carolingien du commentaire ancien sur Martianus, quant à lui, se comporte de manière fort différente. Comme Boèce, il a jugé bon d'introduire ici des illustrations en rapport avec les trois sortes de nombres déficients, abondants et parfaits. Mais la mythologie et le Physiologus sont abandonnés. C'est le christianisme qui est sollicité. La situation du

33. Introduction arithmétique, I, 14, 3.

34. Odyssée, XII, v. 85.

35. Introduction arithmétique, I, 15, 1.

36. Cf. Hésiode, Théogonie, v. 145.

37. Institution arithmétique, I, 19, 8. 
nombre parfait, moyen terme entre l'excès qui caractérise le nombre abondant et le défaut qui caractérise le nombre déficient, est comparée à celle de la justice éternelle entre les deux vices de la justice humaine, qui pèche par excès ou par défaut. "La justice éternelle... est parfaite comme le nombre $6 \ldots$; la justice humaine... trompe très souvent par des jugements soit excessifs, comme le nombre $12 \ldots$, soit inférieurs à ce qu'il faudrait, comme le nombre $16 \ldots »$ : tels sont les éléments importants de ce qu'il écrit ici. Et c'est bien le point le plus original de cette glose.

\section{La justice éternelle}

Avec la justice, c'est un thème d'abord biblique et ensuite chrétien que l'on voit apparaitre dans cette discussion arithmétique. Si le psaume 111, 3 proclame que iustitia eius manet in aeternum, «la justice de Dieu demeure pour l'éternité », la justice " éternelle », thème plus spécifiquement chrétien parce qu'en réalité la justice éternelle de Dieu est le Christ ${ }^{38}$, souvent appelé aussi le "soleil de justice $\aleph^{39}$, est celle qui s'impose à l'esprit de l'auteur du commentaire. Elle est celle qui porte à mépriser les choses du siècle pour mériter la vie éternelle ${ }^{40}$. Parler de la « justice éternelle» et parler de la «justice de Dieu» revient au même ${ }^{41}$. Il est remarquable, du reste, que les occurrences de l'aeterna iustitia (dans cet ordre, plutôt) soient finalement peu nombreuses dans les écrits patristiques ${ }^{42}$. Mais parmi les passages patristiques qui parlent de l'aeterna iustitia, il en est un qui revêt pour nous une importance particulière, parce qu'il présente cette aeterna iustitia comme une medietas, même si ce n'est

38. Jérôme, in Danielem, 3, 9: et manifestata est iustitia aeterna, et unctus est sanctus sanctorum, id est Christus.

39. Jérôme, in Abacuc, 2, 3 : solem iustitiae Christum.

40. Prosper d'Aquitaine, expos. psalm. 118 : qui propter aeternam iustitiam Dei temporalia neglegens uitam sperat aeternam, "celui qui, négligeant à cause de la justice éternelle de Dieu les choses du siècle, espère la vie éternelle. »

41. Cassiodore, expos. psalm. 118 : Iustitia uero Domini... in aeternum seruat, quoniam ipsa semper aeterna est, "Mais la justice du Seigneur... sauve pour l'éternité, parce qu'elle est elle-même toujours éternelle. »

42. L'expression iustitia aeterna apparaît deux fois chez Tertullien, aduersus Iudaeos, VIII ; voir ensuite Augustin, De libero arbitrio, I, 5 ; Lettre 140, 30 : illa qua ipse iustus est, aeterna sua et incommutabili iustitia, "par la justice par laquelle Dieu lui-même est juste, sa justice éternelle et immuable »; Contra Iulianum opus imperfectum, I, 49 ; Isidore, Sententiae, III, col. 653 : malos bic remunerat per temporalem clementiam, et illic punit per aeternam iustitiam, "(Dieu) accorde des biens aux méchants en ce monde à cause de sa clémence qui s'inscrit dans le temps, et les punit dans l'autre à cause de sa justice éternelle »; Grégoire le Grand, Moralia in Iob, XVI, 56 ; Dialogues, IV, 29. 
pas exactement dans le même sens que dans la glose sur le «nombre parfait ». Il s'agit de quelques lignes d'Augustin ${ }^{43}$ :

Quia omnia quae in contrarium pergunt per aliquid medium redducuntur, ab aeterna iustitia temporalis iniquitas nos alienabat, opus ergo erat media iustitia temporali, quae medietas temporalis esset de imis, iusta de summis, atque ita se nec abrumpens a summis et contemperans imis ima redderet summis. Ideo Christus mediator Dei et hominum dictus est, inter Deum inmortalem et hominem mortalem Deus et homo, reconcilians hominem Deo, manens id quod erat, factus quod non erat.

Puisque les choses qui vont dans des directions opposées ne peuvent être rapprochées que par un moyen terme et que l'injustice de ce monde nous éloignait de l'éternelle justice, il nous fallait donc la médiation d'une justice qui tînt à la fois du temps et de l'éternité, de la terre et du ciel, et qui sans rompre avec les choses d'en haut s'accommodât à celles d'en bas, de manière à réunir les unes aux autres. C'est pour cela que le Christ a été appelé médiateur de Dieu et des hommes : Dieu et homme entre Dieu immortel et l'homme mortel, il nous réconcilie avec Dieu, en demeurant ce qu'il était et en devenant ce qu'il n'était pas.

De la doctrine de la justice éternelle intermédiaire entre Dieu et l'homme, on peut garder l'idée de la justice éternelle "intermédiaire », que l'on peut appliquer ensuite au statut de cette justice éternelle par rapport à la justice humaine.

\section{Justice humaine}

En face de la justice éternelle, la justice humaine est souvent citée par la littérature scripturaire et patristique dans un rapport d'opposition complète. Il faut distinguer radicalement justice humaine et justice divine ${ }^{44}$. La justice humaine n'est qu'orgueil ${ }^{45}$ et qu'injustice quand elle est comparée à la justice de Dieu ${ }^{46}$.

43. De consensu euangelistarum, I, 35, 53.

44. Augustin, Contra Iulianum opus imperfectum, III, 27 : ab bumana iustitia discerne dininam.

45. Augustin, Lettre 194, 5 : ... tamquam diceret: audito quod stipendium peccati sit mors, quid te disponis extollere, o bumana non iustitia, sed nomine iustitiae plane superbia? " c'est comme s'il disait : Pourquoi, lorsque tu entends que la mort est la solde du péché, te prépares-tu à t'élever, ô justice humaine qui n'es que de l'orgueil sous le nom de justice ? »

46. Arnobe le Jeune, in psalm. 142 : Omnis enim pulchritudo te praesente deformis est, omnis fortitudo te praesente infirma, omnes diuitiae te praesente mendicitas, omnis iustitia bumana te praesente iniustitia, "Car toute beauté, devant toi, est laideur, et toute force devant toi faiblesse, toute richesse mendicité devant toi, devant toi toute justice humaine est injustice »; cf. Grégoire le Grand, Moralia in Iob V, 37 : Humana iustitia diuinae iustitiae comparata iniustitia est, quia et lucerna in tenebris fulgere cernitur sed in solis radio posita tenebratur. 
La comparaison entre les deux justices apparait véritablement comme un thème caractéristique de Grégoire le Grand dans les Moralia in Iob. À la phrase qui vient d'être citée en note, on peut en ajouter bien d'autres, qui disent la même chose dans les mêmes termes ${ }^{47}$. On est sans doute en droit de soupçonner une influence plus ou moins directe de ce traité de Grégoire le Grand sur le commentateur.

Mais ce lieu commun est reformulé et réutilisé de façon originale par l'auteur du commentaire. Le reproche fait à la justice humaine est arithmétiquement explicité. Écartelée entre le «trop» et le "pas assez », elle manque ainsi aux exigences de la vraie iustitia. Elle partage l'incomplétude ou l'excès des nombres appelés «abondants » et « déficients » dont il est question dans ce passage du commentaire sur Martianus.

\section{Originalité du lexique du commentateur}

La doctrine du nombre "parfait» situé comme un moyen terme entre le nombre "déficient» et le nombre "abondant», avatar, donc, de la vertu intermédiaire entre les vices, si elle est immuable dans les textes anciens où elle est répandue, est exprimée avec des éléments de vocabulaire qui sont susceptibles de variation.

Ici, le commentateur parle de l'excès en le désignant par le mot superfluum, et du défaut en le nommant defectus. Le couple superfluum/defectus manifeste une certaine originalité lexicale dans la mesure où on ne le retrouve pas dans les autres textes arithmétiques comparables et plus anciens. Martianus Capella lui-même, on l'a dit plus haut, n'avait pas ces spéculations sur le vice et la vertu, et on ne les trouve pas non plus, après lui, chez Cassiodore ni chez Isidore, puisque les Étymologies, suivant le modèle cassiodorien, se contentent des définitions stricto sensu de ces trois catégories du nombre pair, sans y ajouter quelque référence morale que ce soit. Chez Boèce, c'étaient les mots de numerositas, de plenitudo, d'abundantia, de paupertas et d'inopia que l'on rencontrait à propos des nombres déficients et abondants, ainsi que le verbe exundare ${ }^{48}$. Pour parler du juste milieu et des excès, Boèce employait les termes temperamentum

47. IX, 18 : Vt enim saepe diximus, omnis humana iustitia iniustitia esse conuincitur, si districte indicetur; XVII, 15 : Humana quippe iustitia auctori comparata, iniustitia est; XXI, 20 : Sciunt quippe quia omnis bumana iustitia iniustitia esse reprehenditur, si dininitus districte indicetur; XXXV, 2 : Omnia enim humana quae iusta, quae pulchra sunt, Dei iustitiae et pulchritudini comparata, nec iusta nec pulchra sunt.

48. Tous ces mots sont dans l'Institution arithmétique, I, 19 : numerositas, plenitudo, paupertas, inopia au $\int 2$; abundantia au $\int 9$; exundare au $\int 4$. 
et intemperantia ${ }^{49}$. Jamais Boèce n'avait recours à superfluum ni à defectus pour faire allusion à la théorie des vices et de la vertu.

On peut étendre la valeur de cette constatation. Pour autant que l'on puisse en juger, le triptyque formé par le superfluum et le defectus encadrant la uirtus n'est pas attesté (on parle de l'expression lexicale) dans la littérature latine classique. Il ne se trouve pas non plus dans la littérature patristique. Il faut attendre l'époque médiévale pour des occurrences de superfluum (ou superfluitas) opposé à defectus (ou defectio) ; et curieusement, les attestations les plus nettes s'en trouvent dans le même texte, qui est la traduction par Jean Scot Érigène des Quaestiones ad Thalassium de Maxime le Confesseur. On y lit que la vertu omnes naturae superfluitates atque defectiones circumcidit, " retranche tous les excès et tous les défauts de la nature $»^{50}$; plus loin, les mêmes quaestiones ${ }^{51}$ marquent une opposition entre per defectum et in superfluis; mais surtout, dans l'explication de la signification des cent vingt mille habitants de Ninive qui « ne distinguent pas leur droite de leur gauche » (Jon 4, 11), le texte $\mathrm{dit}^{52}$ :

Ipsa etenim secundum uerbum scientia uirtutum, id est ipsa per operationem uerae uirtutum causae cognitio, ignorantiam perfecte facere consuenit ipsius ueluti dextrae et sinistrae utrimque accumbentis medietati uirtutum superfluitatis atque defectus.

C'est précisément la connaissance des vertus, entendue au sens propre du mot, c'est-à-dire la connaissance même, dans sa réalité, de la véritable cause des vertus, qui rend parfaite l'ignorance de l'excès et du manque, symbolisés par la droite et la gauche, qui de chaque côté entourent le moyen terme des vertus.

Et quelques lignes plus loin ${ }^{53}$ :

intelligens virtutem, ac per boc neque dextram per superfluitatem neque sinistram per defectum cognoscens.

Comprenant la vertu, et par cela ne connaissant ni la droite, par excès, ni la gauche, par défaut.

Quels que soient les rapports qui apparaissent entre la glose sur le nombre parfait et ces passages de Jean Scot (et l'on ne peut se défendre de les percevoir comme très étroits), le choix des mots ne relève pas du hasard chez le commentateur. Si les différentes appellations latines du

49. Institution arithmétique, I, 19, 9.

50. Maximi Confessoris Quaestiones ad Thalassium, quaestio 40, 1. 68-69 Laga \& Steel 1980.

51. Op. cit. quaestio 56, 1. 144 Laga \& Steel 1990.

52. Ibid. quaestio 64, 1. 309 Laga \& Steel 1990.

53. Ibid. 1. 321. 
nombre « déficient» par rapport au vice par « défaut » ne suggèrent pas ici de remarques intéressantes, il est patent, d'un autre côté, que le commentateur a choisi superfluum pour exprimer l'« excès » parce que le mot superfluus est précisément celui qu'emploient certains textes pour désigner le nombre «abondant». La relation entre «nombre abondant» et « excès » en est soulignée. De fait, Boèce, qui sera suivi par Cassiodore et par Isidore, appelle superflui les nombres «abondants » que Martianus ne désigne que comme ampliores perfecti, alors que le Livre des Nombres isidorien $^{54}$ les appellera plus quam perfecti, comme dans la glose qui nous occupe ici. Parlant du superfluum qui caractérise les nombres superflui, le commentateur s'inscrit donc de manière explicite dans la mouvance de Boèce, dont il a à l'esprit aussi bien les définitions arithmétiques que les élargissements éthiques à propos des nombres parfaits et de ceux qui ne le sont pas.

\section{Traduction de la glose}

Une fois éclairées les différentes questions que nous avons abordées, il est possible de proposer une traduction pour ce texte :

Les philosophes disent que la vertu occupe toujours un juste milieu entre deux vices. Car le vice naît toujours soit de l'excès, soit du défaut. Entre l'excès et le défaut se trouve la perfection; ce qui n'est ni plus, ni moins, occupe le juste milieu. Si en effet la vertu consiste dans le mouvement rationnel de l'âme, il s'ensuit que le vice se trouve dans le mouvement irrationnel. Ainsi donc, ce qui n'atteint pas à la raison parfaite ou qui dépasse la perfection de la raison est un vice. Entre ces deux extrêmes, le mouvement rationnel, précisément, auquel rien n'est ajouté par excès, mais rien non plus n'est retranché, est un moyen terme parfait, et pour cette raison c'est la vertu. Sur ce modèle, donc, on dit que le nombre parfait possède la ressemblance avec la vertu, tandis que le plus-que-parfait et le moins-que-parfait sont des images du vice. Par exemple, la justice éternelle, de même que l'on ne sert que Dieu seul ${ }^{55}$, est parfaite, ne

54. Liber numerorum, 13, 65.

55. Il y a ici, semble-t-il, une difficulté de texte. Les deux manuscrits de Leyde et de Besançon portent la leçon quasi soli Deo seruitur, littéralement «de même que l'on ne sert que Dieu seul », que nous gardons ici mais dont le sens parait peu satisfaisant. Quelles corrections pourrait-on suggérer? L'expression au datif soli Deo est naturellement fréquente dans les textes patristiques. Avec le verbe seruire, soli Deo seruire se trouve par exemple chez Cyprien, ad Fortunatum, 11 ; mais soli Deo seruiendum est relativement fréquent. L'origine est la phrase scripturaire Dominum Deum tuum adorabis et illi soli servies (Dt 6, 13, repris en Mt 4, $10=\mathrm{Lc} 4,8$ ), souvent citée et commentée par les Pères. Si l'on garde, dans la présente glose, les mots soli Deo seruitur, il est nécessaire de corriger quasi en qua, pour obtenir «la justice éternelle, par laquelle on sert Dieu seul»; on pourrait aussi 
contient rien en excès, n'a aucune imperfection, de même que le nombre six, qui est égal à ses parties; tandis que la justice humaine, qui trompe très souvent, ou bien juge par excès, comme dans le nombre douze, que ses parties surpassent, ou bien juge moins qu'il ne faut, comme dans le nombre seize, auquel ses parties ne sont pas égales, car elles produisent un nombre plus petit, à savoir quinze.

\section{Synthèse}

Le socle ancien de l'édifice est visible et bien conservé. Il s'agit de la théorie des nombres parfaits opposés aux nombres déficients et abondants. Même si l'on ne possède de traces de cette théorie qu'à partir de Nicomaque et de Théon (c'est-à-dire à partir du IIe s. apr. J.-C.), on a les meilleures raisons d'en faire remonter les origines aux pythagoriciens eux-mêmes.

Les exemples canoniques sont toujours là, ayant traversé les siècles depuis Nicomaque, en grec et en latin : le nombre 6 est le prototype du nombre parfait, le nombre 12 le modèle du nombre abondant, et le nombre 16 celui du nombre déficient. Ils sont exposés en des termes qui ont peu varié depuis Nicomaque, en grec, et Boèce, en latin.

L'élément essentiel de variation consiste dans la suppression des illustrations mythologiques qui accompagnaient chacun des deux nombres déficient et abondant, et dans l'introduction d'un triptyque « justice éternelle // justice humaine par défaut / justice humaine par excès » en parallèle au triptyque «nombre parfait // nombre déficient / nombre abondant ». De l'exemplarité mythologique on passe à l'affirmation théologique. C'est là que se situe le changement de monde. Le texte de Martianus est introduit dans une atmosphère médiévale et chrétienne. Les trois espèces du nombre pair sont illustrées non plus par des curiosités tirées d'un magasin d'antiquités, mais par l'évocation d'une entité éthique encadrée de ses avatars, qui pose, ne serait-ce que sous le texte, la grande question : «Qu'est-ce que la justice ? » et qui, bien avant Pascal mais avec un esprit tout aussi libre, ne craint pas de dénoncer les limites de la justice des hommes en des termes beaucoup plus précis que ne le

corriger quasi en quae et seruitur en seruatur pour obtenir « la justice éternelle, qui est réservée à Dieu seul »; cette correction pourrait se recommander d'un parallélisme avec Jérôme, Contre les Pélagiens, 3, 12 : perpetuitatem autem impeccantiae soli reseruari Deo; en tout cas le problème a été senti par Rémi d'Auxerre qui, dans le passage correspondant de son commentaire sur Martianus, p. 205, 1. 24 Lutz, écrit seruitus au lieu de seruitur, obtenant ainsi « qui est comme une soumission à Dieu seul». 
faisaient les passages patristiques (ainsi ceux de Grégoire le Grand) dont l'auteur, peut-être, tira pour partie son inspiration.

L'enseignement arithmétique en est transfiguré et sublimé. Certes, demeure la doctrine du nombre parfait, impliquée par le texte de Martianus. Mais on se demande désormais à bon droit si le but du rédacteur a été d'apporter sur ce point des éclaircissements au texte de Martianus, ou (plutôt ?) d'utiliser le prétexte offert par la thématique du nombre parfait pour introduire à des réflexions morales d'une plus ample portée. Sous ces quelques lignes, on sent un esprit indépendant, capable de synthétiser les enseignements anciens mais aussi de les replacer et de les réutiliser dans une perspective renouvelée. Celui qui écrit ces lignes est un intellectuel étranger au conformisme. L'extraordinaire similitude de son vocabulaire et de celui de Jean Scot Érigène pourrait faire penser que tous deux ne font qu'un seul et même personnage, si la quaestio uexata de la paternité du commentaire ancien sur Martianus n'avait été réglée (provisoirement ?) par son attribution à un auctor incertus qui, pour être proche des milieux irlandais, ne serait ni Dunchad, ni Jean Scot, ni Martin, et si du reste on ne possédait pas, de Jean Scot lui-même, un commentaire du livre VII de Martianus dont le contenu est assez nettement différent de celui des gloses dont nous parlons ici. Il reste que le milieu intellectuel désigné est celui dans lequel évoluèrent des esprits comme celui de l'Érigène.

\section{Conclusion}

Quoi qu'il en soit, on a ici un exemple intéressant de la fécondité de la tradition de la spéculation philosophique d'origine grecque sur les êtres mathématiques. L'auteur du texte devenu glose sur le nombre parfait travaille en effet sur la base fournie par l'ancienne spéculation pythagoricienne. Mais il se met, d'entrée de jeu, sous la protection des auteurs latins car, si l'expression initiale philosophi dicunt peut sembler renvoyer seulement de façon vague à un enseignement effectivement très répandu, il se pourrait aussi qu'elle fasse allusion précisément aux écrits de Boèce lui-même ${ }^{56}$; et il se replace aussi dans la ligne du néoplatonisme latin avec son enseignement sur les deux mouvements de l'âme, «rationnel » ou « régulier» (rationalis), et «irrationnel » ou « irrégulier» (irrationalis). Ce néoplatonisme doit être concilié avec le christianisme, dont la marque se lit dans certaines expressions qui apparaissent comme des réminiscences de passages patristiques. La perfection numérique continue à porter

56. De fait, dans la même première phrase, l'expression uirtutem medietatem... tenere fait penser à ce que Boèce écrivait dans le De persona et naturis duabus, PL 64, col. 1352 C : ... uirtutes medium tenent... Medietatem igitur uirtus tenet. 
notre auteur à la recherche de correspondances, mais celles-ci sont cosmiques, théologiques et chrétiennes, non plus mythologiques et érudites, et la réflexion culmine non pas sur l'évocation du Cyclope ou de l'Hécatonchire, mais sur des considérations essentielles à propos de la justice. Nombre parfait, vice et vertu, élans de l'âme, justice, sont mis en cohérence dans une synthèse typique sans doute de la mentalité des intellectuels médiévaux, qui s'appuie sur des données anciennes, mathématiques et philosophiques, mais les reformule, les transfigure et les sublime. 


\section{BIBLIOGRAPHIE}

HeAth, Th. L. 1921 : A History of Greek Mathematics, Oxford, 2 vol., 1921 (nombreuses réimpressions).

Hiller, E. Theonis Smyrnaei philosophi platonici Expositio rerum mathematicarum ad legendum Platonem utilium, recensuit -, Leipzig, 1878 (Bibliotheca scriptorum graecorum et romanorum teubneriana).

LAGA, C. \& C. STEel 1980 : Maximi Confessoris Quaestiones ad Thalassium, I : Quaestiones I$L V$, una cum latina interpretatione Joannis Scotti Eringenae juxta posita ed. —, Turnhout, 1980 (Corpus christianorum. Series graeca, 7).

- 1990 : Maximi Confessoris Quaestiones ad Thalassium, II : Quaestiones LVI-LXV, una cum latina interpretatione Joannis Scotti Eringenae juxta posita ed. —, Turnhout, 1990 (Corpus christianorum. Series graeca, 22).

Michel, P.-H. 1950 : De Pythagore à Euclide: contribution à l'bistoire des mathématiques préeuclidiennes, Paris, 1950.

PrÉAUX, J. G. 1953 : «Le commentaire de Martin de Laon sur l'œuvre de Martianus Capella », Latomus, 12 (1953), p. 437-459. 